\title{
Research on characteristics of Chinese language in the presentation of "Face"
}

\author{
Lifang Liu* \\ School of Urban Culture, South China Normal University, Foshan, Guangdong, China
}

\begin{abstract}
: with the development of society, interpersonal relation has become more and more complicated. "Face" has been defined as "public self-image" and occupies a very important position in interpersonal communication. Therefore, this thesis mainly studies the characteristics of Chinese language in the presentation of "face". At first, it referred to current situation of face studies overseas. It can be seen that the "defense-protect" face work theory proposed by Goffman is the important foundation for face problem studies under different angles in academic world. Then, it discussed identity theory based on social psychology in combination of our traditional culture. At last, it selected 85 groups of effective linguistic data according to characteristics of Chinese language; and studied face work. Moreover, it conducted questionnaire survey in combination of face work theory. Thus, it obtained the core factors of face and other important conclusions.
\end{abstract}

Keywords: face relation; social psychology; identity theory; linguistic data

\section{INTRODUCTION}

Back in 1980s, Brown proposed the relational normal form of face and politeness. Face was defined as "public self-image". When people's face is threatened, they will take measures to reduce the threat. According to face work, this thesis firstly referred to current situation of foreign studies of face. It can be known that the "defense-protect" face work theory proposed by Goffman is the important foundation for the academic circles to study face issues in different angles. Then, in combination of our traditional culture, this thesis discussed identity theory based on social psychology. According to current situation of our research on face work, many predecessors have made great contribution, among which:

In 2014, Yanhui Liu explained detailed connotation of face theory in Analysis of International Business Negotiation Interpretation Based on Face Theory. She introduced the important principles which interpreters shall follow in international business negotiation. In addition, she combined several interpretation examples for demonstration. At last, she concluded how interpreters shall take measures to follow face theory and facilitate negotiation in international business negotiation ${ }^{[1]}$.

In 2012, Liuyang Chen studied the linguistic data of talk conflicts with genetic relationship in Research on Genetic Relationship Talk Conflicts Based on Face Theory. He disclosed the construction, types, and reasons of talk conflicts with generic relationship. He has enriched the research on talk conflict and can further provide reference guidance for talk analysis. At last, he concluded the relation between talk conflict and face. It can be known that there is both compatibility and differentiation between western face theory and our generic relationship ${ }^{[2]}$.

In 2012, Lichao Yin explained the importance of combining face and wedding ceremony by analyzing the integrating points between these two in Research on Rural Wedding Ceremony in Perspective of Face Theory. She pointed out that face behaviors in our weddings are in interposed "narration form" which takes family as the core. There's no proportion between behavior strength and family economy. Face behavior strength in wedding is a kind of yielded mental states in face theory ${ }^{[3]}$.

In 2013, Jing Li made detailed studies of Chinese and American negotiation styles from perspective of face theory in Research on Chinese and American Negotiators' Negotiation Styles in Perspectives of Face Theory and Politeness Theory. He found that China is a country full of face recognition. When there's any conflict between benefit and face, Chinese 
negotiators tend to put face in the first place. The higher status a Chinese negotiator owns, the more he/she will maintain face in negotiation ${ }^{[4]}$.

Based on the predecessors' studies, this thesis selected 85 groups of effective linguistic data in combination of our traditional culture and the identity theory based on social psychology. It studied face work and made questionnaire survey according to face work theory. As a result, this thesis has summarized the core factors of face and reached other important conclusions, aiming to provide significant contribution to the research on characteristics of Chinese language and on interpersonal communication.

\section{RESEARCH ON FACE WORK}

The concepts of face and face work were firstly proposed by American sociologist Goffman in the 1950s. Goffman defined face work as a series of activities related to face behaviors. As a result, once an individual of face is threatened in communication, face work comes. Goffman applied bisection method in face work. He divided behaviors in face work into two types: one is to protect face and the other is to save face. Generally, we call it the "defense-protect" face work which has become the important foundation for the academic circles to study face issues in different angles. This thesis is no exception. Besides, Goffman made "amendment and improvement" analysis: if one's face is threatened, he/she will try hard to amend and improve the relation to save face.

Goffman also combined theory of drama and used one's changes on the stage and behind the scene as metaphor of people's face work. He pointed out that face contains dynamic nature which means face is earned through mutual negotiation in face work. Besides, face contains sociality which means face is obtained through others' assessment on one person in a group. Therefore, normal and smooth face work also requires mutual respect between the two communicators. Goffman once tried to distinguish the protection positioning of other's face and the defense positioning of one's own face during face work. However, he found these two parts are indivisible in fact. It can be known from Figure 1 for the "defense-protect" face work pattern as shown below that other's face protection and our own face defense are within a community of correlative dependence.

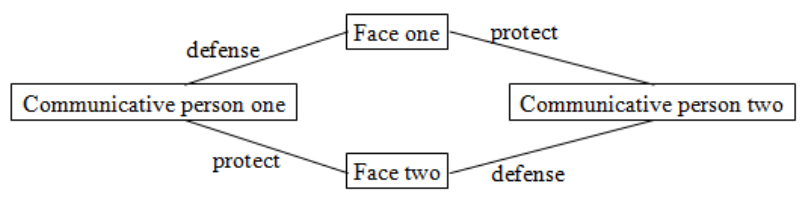

Figure 1. "Defense-protect" face work pattern

According to Figure 1, communicator 1 will start defense mode for face 1 during actual communication. In the meantime, communicator 2 will take protective measures for face 1 . Similarly, same pattern will be applied for face 2 as shown in Figure 1. Thus, interpersonal communication is orderly and harmonious for sustainable and circulatory development.

\subsection{Face work studies conducted by our scholars}

Different from foreign scholars, our scholars have studied problems in face work from perspective of sociology among which the "favor-face" pattern established by Guangguo Huang as shown in Figure 2 has been widely recognized.

Based on the unique folk culture habits of our country and the importance of favor in face work, Guangguo Huang established a reasonable framework to explain people's behaviors in face work. He proposed impression management behavior which is the so-called "face work". He thinks many behaviors in face work are made on purpose to leave good impression on others. In combination of the viewpoints about favor and face shown in Figure 2, we can find that face work is not only individual's behavior; however, it can also represent one's social status and reputation.

\section{IDENTITY THEORY BASED ON SOCIAL PSYCHOLOGY}

According to current situation of our research on face

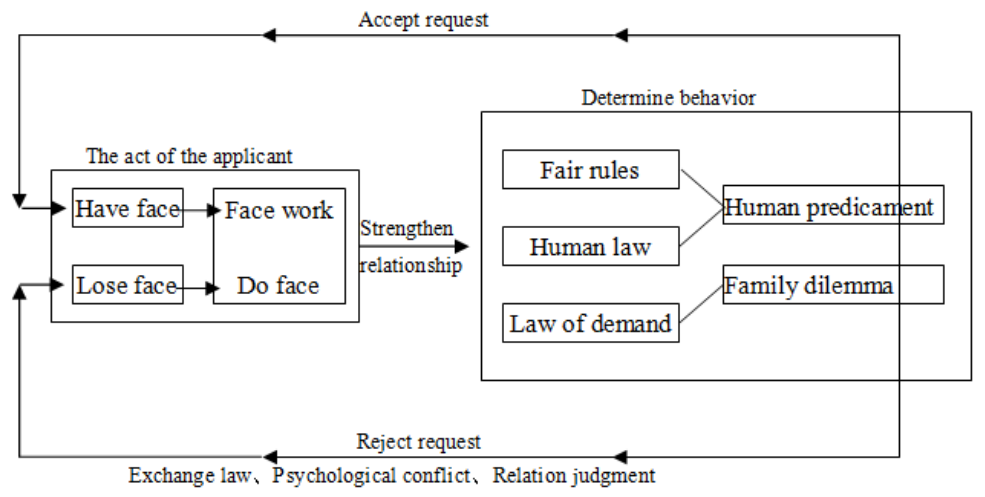

Figure 2. Theory patterns of favor and face 
work, it can be known that there's close relation between face work and identity theory. Identity theory is an emerging social psychological theory. Most scholars agree on the non-qualitative nature of identity, meaning identity is a series of self-configured and self-explained identity assemblies instead of given ones. As a complex collection with multi levels, modern society is full of individuals who share distinct differences with each other while have correlative dependence at the same time. Therefore, identity is always regarded as a set of internalized character expectation. During the process of face work, ideal self-identity can be built in front of others through self-explanation. This process can't be separated with internalized self-recognition and external circulation made by others. See Figure 3 for the relation between identity and face.

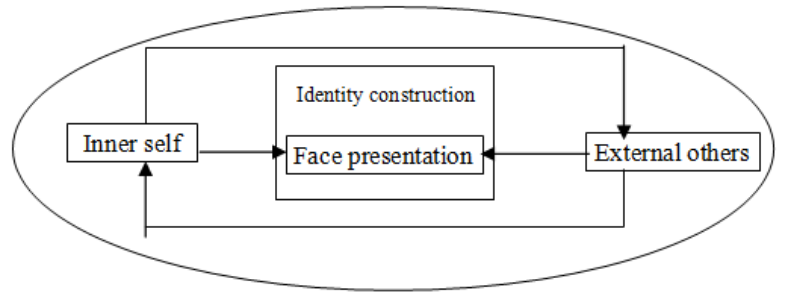

Figure 3. Identity-face relations

\subsection{Research framework of this thesis}

According to the identity theory, face, and analysis of face work theory described above, this thesis will discuss the language characteristics of Chinese culture in the following step. At first, see Figure 4 for the research theory framework applied in this thesis:

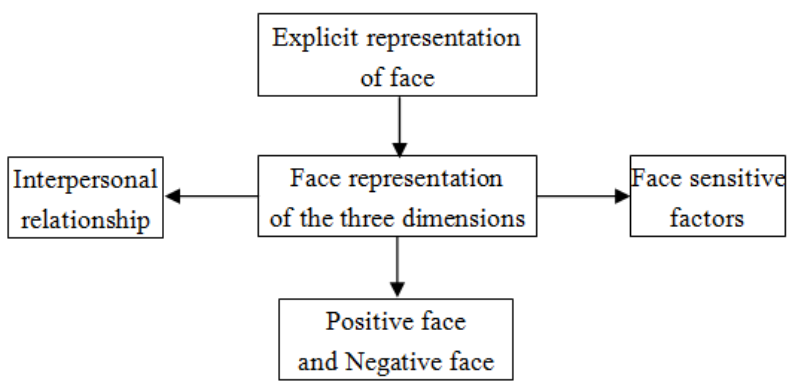

Figure 4. Theory framework of research on characteristics of Chinese language under the presentation of face study

As shown in Figure 4, according to the interpersonal relations reflected in "face", this thesis conducted multi-dimension analysis combing he self-presentation in identity theory and from perspective of face sensibility. In combination of our traditional culture, it can be known that Chinese tend to present face in communication. Therefore, this thesis took the explicit presentation of face as analysis subject. It has obtained the explicit expression forms of face and further studied the characteristics of Chinese in view of "face" presentation by applying the audio media language text library retrieval system of Communication University of China.

\section{DATA DESCRIPTION AND RESEARCH ON CHARACTERISTICS OF CHINESE LANGUAGE}

\subsection{Research on description of linguistic data}

At first, this thesis selected 85 groups of effective linguistic data. By applying FCA, it can be seen that there's close relation between the presentation of face in Chinese and social factors. In a large number of explicit topics about face, "moral quality, "favor" and "dignity" stood out. Topics about moral quality occurred in 27 groups of dialogue. Topics about favor occurred in 26 groups of dialogue. Topics about dignity occurred in 20 groups of dialogue. These three topics occupied $31.8 \%, 30.6 \%$ and $23.5 \%$ of the total sum respectively while the rest topics only occupied $14.1 \%$. See the relation between topic about moral quality and prominent social cultural factors and social identity characteristics in Figure 5 as shown below:

According to Figure 5, it can be known that the social cultural factors mainly related to topics about moral quality are social status, educational status, and occupation, involving each kind of interpersonal relations and communicators at all ages. Moreover, topics about favor occupied $30.6 \%$ which ranked only second to topics about moral quality. See Figure 6 as shown below for the relations between favor and each social cultural factor.

Topics about dignity occupied $23.5 \%$. See Figure 7 as shown below for the relations between dignity and each social cultural factor.

Figure 5, Figure 6 and Figure 7 described above clearly manifest the relations among face presentation, social culture, and communication individual.

\subsection{Research on description of questionnaire data}

In combination of the social psychology theory described in Section 3, the author designed questionnaires detailing the definition of face under linguistic context of Chinese. It can be seen by summarizing that the testees tend to use "dignity", "favor", "reputation", "honor" and "self-esteem" to define face. See Table 1 for the occurrence frequency of each word as follows:

According to Table 1, it can be known that dignity, reputation and honor had high frequency of occurrence. Responders of the questionnaires defined these three words as the keywords of face, constituting a great difference from those of western "self-image".

Moreover, based on the "defense-protect" face work theory; the questionnaires listed 18 influence factors which may arouse communicator's psychological attention to face behaviors. In combination of the 


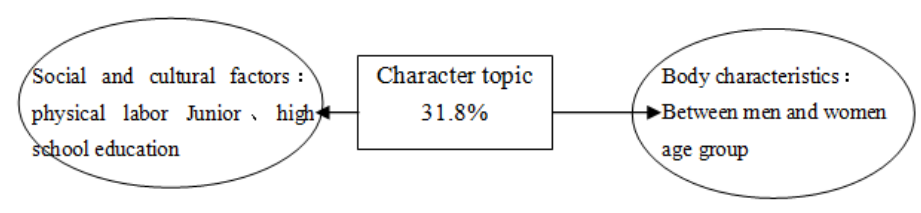

Figure 5. Correlations between moral quality and social cultural factors and identity characteristics

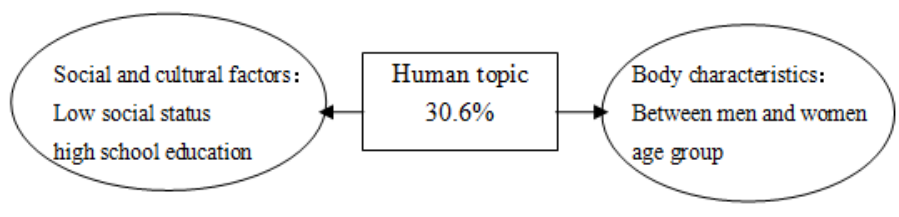

Figure 6. Correlations between favor and social cultural factors and identity characteristics

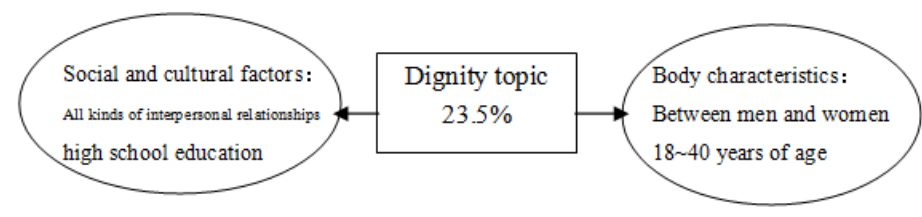

Figure 7. Correlations between dignity and social cultural factors and identity characteristics

Table 1. Perception of face

\begin{tabular}{llll}
\hline $\begin{array}{l}\text { Keywords involved in conception } \\
\text { of face }\end{array}$ & Frequency of occurrence & $\begin{array}{l}\text { Keywords involved in conception } \\
\text { of face }\end{array}$ & Frequency of occurrence \\
\hline Dignity & $20.5 \%$ & Reputation & $13.1 \%$ \\
Sensibilities & $10.7 \%$ & Honor & $9.5 \%$ \\
Respect & $6 \%$ & Ability & $6 \%$ \\
Status & $6 \%$ & Achievement & $6 \%$ \\
Occasion & $3.6 \%$ & Image & $4.8 \%$ \\
Accomplishment & $2.4 \%$ & Self-esteem & $9.5 \%$ \\
Social identity & $1.2 \%$ & Emotion & $1.2 \%$ \\
\hline
\end{tabular}

five-level evaluation on the 18 factors, the core factors constituting face are shown in Figure 8.

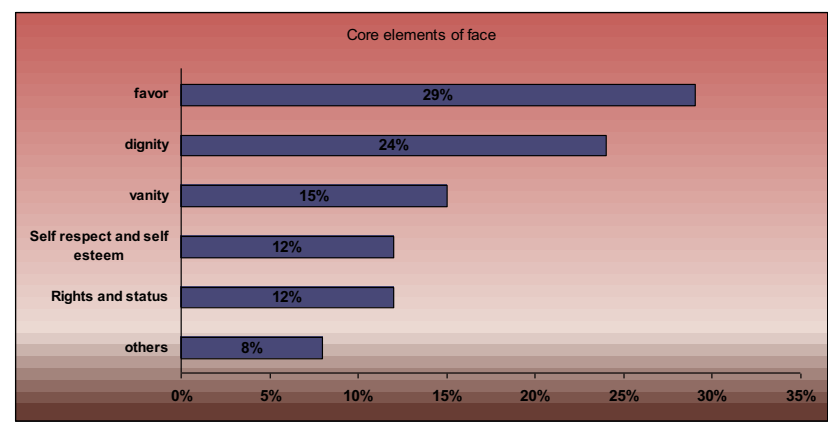

Figure 8. Core factors constituting face

According to Figure 8, it can be known that Chinese understanding of face mainly involve "favor", "dignity", "vanity", "self-abasement and self-esteem" and "rights and status". These five concepts constitute the core factors of face. In the following step, this paper made statistical analysis of the perception parts in face factors and further designed questionnaires. It divided the rating sections into five levels -5 scores, 4 scores, 3 scores, 2 scores and 1 score-corresponding to "have face very much", "have face", "lose face", "lose face comparatively" and "lose face badly". See Figure 9 as shown below for the statistical results.

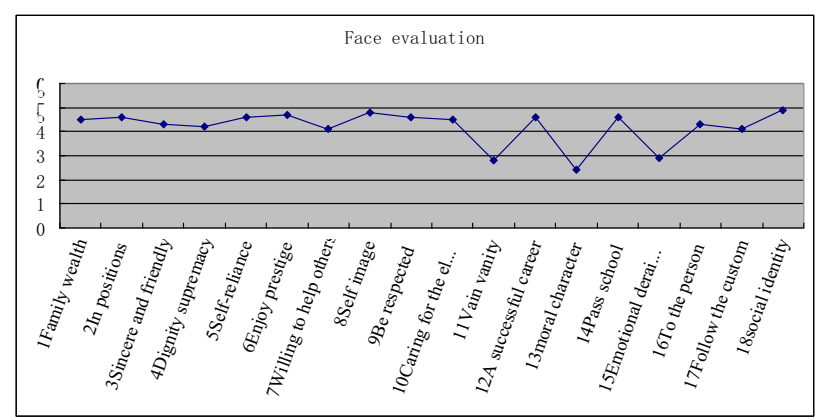

Figure 9. Evaluation on perception degree of face

According to Figure 9, it can be seen that all the other 15 factors except No.11, No.13 and No.15 factors rated 4 to 5 scores. Thus, it can be known that there's no significant difference among each factor. 
Hypocrisy and vanity, moral trait, and emotional derailment were in low-score sections, showing the testees think these three factors will make them lose face badly.

\section{CONCLUSIONS}

This thesis selected 85 groups of effective linguistic data. According to research, it can be found that there's close relation between the presentation of face in contemporary Chinese and social factors. In a large number of explicit topics about face, "moral quality, "favor" and "dignity" stood out. Topics about moral quality occurred in 27 groups of dialogue. Topics about favor occurred in 26 groups of dialogue. Topics about dignity occurred in 20 groups of dialogue. These three topics occupied $31.8 \%, 30.6 \%$ and $23.5 \%$ of the total sum respectively while the rest topics only occupied $14.1 \%$.

By compiling and issuing questionnaires, this thesis listed 18 influence factors which may arouse communicator's psychological attention to face behaviors based on the foundation of "defense-protect" face work theory. In combination of the testees' five-level evaluation on the 18 factors, it can be concluded that Chinese understanding on face mainly involve "favor", "dignity", "vanity", "self-abasement and self-esteem" and "rights and status". All these concepts constitute the core factors of face.

\section{REFERENCES}

[1] Liu, Y.H. 2014. Analysis of International Business Negotiation Interpretation Based on Face Theory. Shandong Normal University.
[2] Chen, L.Y. 2012. Research on Genetic Relationship Talk Conflicts Based on Face Theory. Bohai University.

[3] Yin, L.C. 2012. Research on Rural Wedding Ceremony in Perspective of Face Theory. Harbin Engineering University.

[4] Li, J. 2013. Research on Chinese and American Negotiators' Negotiation Styles in Perspectives of Face Theory and Politeness Theory. Tianjin University of Commerce.

[5] Zhao, Z.J. 2009. Study of the Impact of Interpersonal Conflict Inside a Team and Face Problems Have on Team Creativity. Zhejiang University.

[6] Chen, Q. 2010. Application of Face Theory in Classroom Contradiction. East China Normal University.

[7] Chen, W.P. 2013. Exploration of Employee's Structure of Face Theory within an Organization and Its Influence on Suggestion and Silence Behavior. Suzhou University.

[8] Gong, W.B. 2013. Compilation and Feature Research on Questionnaire of Undergraduate's Face Theory. Southwest University.

[9] Mao, S.S. 2009. Explanation of Face Phenomena in Chinese Culture Based on Identity Theory. Northeast Normal University.

[10] Yan, B.Y. 2008. Application of Face Strategy in Oral English. Jilin University.

[11] Hu, C.S. \& Chi, Y.C. 2012. Puzzle of feedback bargain, investor's sentiment and volatility. South China Journal of Economics, 3: 37-48.

[12] Zhao, Z.J. 2012. Face theory research summary. Journal of Chongqing University (Social Science Edition), 05: 128-137.

[13] Hao, Y.X. 2006. Application of face theory in college English class interaction between teachers and students. Journal of Hohai University (Philosophy and Social Science Edition), 03: 54-57+94.

[14] Qian, H.Y. 2005. Realization of face theory in English to Chinese appellation wording. Journal of Huizhou University (Social Science Edition), 04: 49-52.

[15] Feng, G.Y. 2007. Comparison between "Face Theory" and English to Chinese face maintaining strategy. Neijiang Science, 04:15-16. 